\title{
La adaptación y las instituciones de educación superior: conceptos y desafíos
}

\author{
Patricia Roncancio-Roncancio
}

\section{RESUMEN}

Este trabajo busca delimitar el concepto de adaptación para configurar un escenario que permita comprender las interacciones entre el concepto y las prácticas administrativas en las instituciones de educación superior (IES) privadas. Con frecuencia se asigna a la administración la tarea de lograr la adaptación de la organización. Sin embargo, no se encuentran trabajos que indiquen cómo el adoptar una noción de la adaptación puede impactar la toma de decisiones y la construcción de indicadores de gestión de las IES. La revisión sistemática de literatura evidencia que los fenómenos adaptativos y administrativos suelen abordarse de manera independiente, por lo que el estado de la teoría en este campo es insuficiente. La ruta metodológica responde a las características de una heurística que posibilite la construcción de un campo de problemas para la investigación y la comprensión de la adaptación de las universidades latinoamericanas, desde su administración. La discusión permite delinear escenarios para la investigación y señala desafíos a nivel de la elaboración de políticas públicas y de la administración de las IES.

Palabras clave: adaptación social, instituciones de educación superior, estudios sociales, administración, universidades privadas. 


\section{A adaptação e as instituições de educação superior: conceitos e desafios para a administração}

\section{RESUMO}

Este trabalho busca delimitar o conceito de adaptação para configurar um cenário que permita compreender as interações entre o conceito e as práticas administrativas nas instituições de educação superior (IES) privadas. Com frequência se designa à administração a tarefa de conseguir a adaptação da organização, porém, não se encontram trabalhos que indiquem como o fato de adotar uma noção da adaptação pode impactar na toma de decisões e na construção de indicadores de gestão das IES. A revisão sistemática de literatura evidencia que os fenómenos adaptativos e administrativos acostumam ser abordados de maneira independente, porque o estado da teoria neste campo é insuficiente. O trajeto metodológico responde às características de uma heurística que possibilite a construção de um campo de problemas para a pesquisa e a compreensão da adaptação das universidades latino-americanas, desde sua administração. A discussão permite definir cenários para a pesquisa e mostra desafios a nível da elaboração de políticas públicas e da administração das IES.

Palavras chave: adaptação social, instituições de educação superior, estudos sociais, administração, universidades privadas.

\section{Adaptation and higher education institutions: concepts and challenges for management}

\section{ABSTRACT}

This work aims to delimit the concept of adaptation in order to shape a stage that allows to understand the interactions between the concept and the administrative practices in private higher education institutions (HEI). The management is often assigned the task of achieving the adaptation of the organization; however, no work can be found that indicates clearly how adopting a notion of adaptation can impact on decision making and on the construction of management indicators in HEIs. The systematic review of the literature shows that adaptive and administrative phenomena are usually addressed independently, so the state of theory in this field is insufficient. The methodological route responds to the characteristics of a heuretics that makes possible the construction of a field of problems for research and the understanding of the adaptation of Latin American universities, from the perspective of their management. The discussion allows delineating stages for research and points out challenges at the level of the elaboration of public policies and of the HEIs' management.

Key words: social adaptation, higher education institutions, social studies, management, private universities. 


\section{Introducción}

La universidad es una institución que conjuga la administración de los recursos generales de cualquier organización con los específicos propios de su naturaleza educativa. Es la manera como se administran los segundos lo que define su identidad (Borrero, 1984). La forma como interactúan y se complementan los aspectos generales y específicos de la administración de las instituciones de educación superior (IES) puede determinar su rumbo y la calidad del servicio educativo.

En el ejercicio de las funciones de dirección y de gestión, la administración tramita acciones para responder a los requerimientos normativos del Estado y coordina la articulación de los procesos académicos y administrativos de la organización. Por consiguiente, mediante sus decisiones y acciones la administración impacta, a través de las funciones sustantivas de la universidad, las relaciones con los entornos, con el Estado y con la sociedad. El intercambio y las relaciones con los actores internos y externos incide en la forma como las IES afectan y son afectadas por las demandas sociales, económicas, ambientales y políticas características del mundo interconectado.

Si bien hay un notable interés y trabajos sobre la administración de las IES, la revisión de literatura específica mostró la carencia de estudios que profundicen en los conceptos y las interacciones entre la administración y la adaptación en el contexto de las universidades privadas iberoamericanas. El estado de la teoría que incorpora los conceptos estructurantes del estudio es "inmaduro" (Merriam, 1988). El estado del conocimiento determina la opción metodológica para precisar el concepto de adaptación y configurar un escenario que permita comprender las interacciones entre el concepto y las prácticas administrativas en las IES privadas.

\section{Método}

El problema es un hecho no resuelto que debe encontrar una respuesta teórica o práctica que posibilite su solución total o parcial (Cerda, 1991:139). En este caso es la búsqueda de una respuesta desde lo teórico para interpretar, comprender y poder intervenir en la práctica y en las prácticas de la administración de las organizaciones de educación superior.

El punto de vista heurístico ha sido tan valioso en ciencias sociales como en las ciencias básicas, como recurso para abordar, comprender y construir problemas encaminados a responder preguntas novedosas para diferentes campos de las ciencias. Además, consecuente con el papel que se asigna a la teoría en este tipo de estudios, la utilización de la técnica de revisión sistemática (Centro Cochrane Iberoamericano, 2012) para focalizar y priorizar, permitió evitar los planos generales y concentrar el trabajo en los aspectos directamente relacionados con el objetivo. La revisión sistemática posibilitó descartar documentos e identificar enfoques y posibles integraciones del concepto de adaptación desde las ciencias biológicas y sociales. En consecuencia, no es un estado del arte como pudiera esperarse en un estudio documental y tampoco tiene la amplitud de un trabajo de corte monográfico.

A diferencia de la pretendida objetividad de los métodos cuantitativos arraigados en el positivismo, la perspectiva heurística admite el involucramiento creativo del investigador (Moustakas, 1990). Esta exploración, orientada hacia la comprensión mediante "la perspectiva invertida", involucra un conjunto de procesos como el pragmatismo. Además de la comprensión, la perspectiva pragmática apunta a la construcción de instrumentos conceptuales y técnicos para resolver problemas (Cherryholmes, 1992; Rossman y Wilson, 1985; Salk, 1983).

Hernández, Fernández y Baptista (2006) subrayan algunos factores que determinan que una investigación inicie como exploratoria, descriptiva, correlacional o explicativa. La investigación deberá iniciarse como exploratoria: a) cuando la revisión de la literatura muestra que no hay antecedentes sobre el tema en cuestión o que, si los hubiera, no son aplicables al 
contexto en el que se desarrolla el estudio; b) cuando las ideas relacionadas con la investigación son vagas; c) el tema se puede haber estudiado, pero con sentidos diferentes. En este punto, es evidente que para articular de manera coherente y legítima los procesos administrativos y de adaptación de las IES es necesario partir de una revisión y precisión conceptual. A partir de los hallazgos en esta fase se pueden construir problemas y escenarios para investigaciones descriptivas, correlacionales y explicativas.

\section{Contexto}

Desde una perspectiva biológica la noción de adaptación está anclada en los principios de la evolución y su meta final es la prolongación, la supervivencia y perdurabilidad de las especies. En la tradición positiva y funcionalista ha sido evidente la tendencia a utilizar conceptos biológicos para explicar y comprender fenómenos sociales. En el caso de la adaptación encontramos una noción con limitaciones al intentar aplicarla a formas de organización cuyos actores e interacciones son de naturaleza fundamentalmente social.

En efecto, la tendencia a explicar la adaptación de las organizaciones a partir de las metáforas biológicas predominó durante buena parte del siglo XX. Transcurridos los tres primeros quinquenios del siglo XXI, la revisión de la literatura muestra el predominio de estudios que se ocupan de la adaptación de los individuos y los equipos de trabajo, y evidencia la insuficiencia y las limitaciones de trabajos que articulen y profundicen las relaciones de la administración con los procesos de adaptación de las universidades en tanto IES.

La revisión muestra que frecuentemente los temas se abordan de manera aislada o las referencias a dichas interacciones son tangenciales e indirectas. Así mismo, adaptación y adaptabilidad suelen utilizarse en un mismo sentido, o lo uno se define en función de lo otro. Otros trabajos se refieren a las capacidades adaptativas como conjunto de procesos y competencias que se requieren para lograr la adaptación de las organizaciones, y otra tendencia que proviene de los estudios del aprendizaje organizacional, vincula la adaptación con los procesos de conocimiento en las organizaciones (Eichholz, 2015).

Más que establecer explicaciones causales entre variables, esta presentación permite explorar interacciones entre la administración general y de las funciones misionales de las IES con factores que podrían aportar o interferir con los procesos de adaptación de las universidades. Para lograrlo, es relevante reconocer la forma como desde diversas disciplinas y enfoques se han llevado a cabo las aproximaciones al concepto, lo que permitiría construir problemas que mediante la investigación pudieran generar metodologías e instrumentos para la comprensión y la resolución de los fenómenos que afectan a las universidades, particularmente de América Latina.

\section{De las metáforas biológicas a los conceptos sociales}

Las reacciones a la hegemonía teórica y metodológica del positivismo y sus aplicaciones en el funcionalismo caracterizaron los desarrollos de las ciencias sociales durante el siglo XX. Superada la fase reactiva fue posible empezar a construir y desarrollar conceptos sociales para comprender y explicar fenómenos sociales.

Dos corrientes dominaron el siglo XX y de ellas emergieron los paradigmas posmodernos de finales de la época. De un lado, las corrientes derivadas de los postulados marxistas y weberianos que asumen la sociedad a partir de la dominación y el conflicto entre grupos sociales. De esta vertiente se desarrollan las teorías de las escuelas críticas que dan un lugar relevante a la comunicación y los conflictos de interés. Por otro lado, el estructural funcionalismo o funcionalismo estructural, que finalizada la primera Guerra Mundial alcanza desarrollos significativos para comprender las estructuras en relación con las funciones sociales de las organizaciones. 
Para resolver las oposiciones planteadas por los enfoques basados en el conflicto social, el estructural funcionalismo parte de la concepción de la sociedad como totalidad, un sistema complejo cuyos componentes en conjunto buscan la estabilidad. Este paradigma propone que a cada estructura corresponde una función y las conductas de los sujetos son determinadas por las estructuras en las que se encuentran inmersos.

En este marco, son pioneros los trabajos de Parsons (1951) con el desarrollo del "Modelo de Intercambio" en el cual se incorpora, de manera explícita, el concepto de adaptación. Este modelo es representativo de las tendencias del momento, en el ámbito de la sociología.

A partir de una concepción jerarquizada de la sociedad, la propuesta sistémica del estructural funcionalismo resalta la influencia que sobre cada sistema tienen las variaciones que ocurren en el sistema superior, determinadas por la apertura a las interacciones entre ellos. Esta visión, que sintetiza Parsons en el "Modelo de Intercambio AGIL", incluye cuatro subsistemas: adaptación, capacidad para alcanzar metas, integración y estado de latencia. La adaptación es la dimensión que representa las fuerzas materiales, condicionantes, restrictivas, aquello que hay que enfrentar y asumir. La economía es la esfera más estrechamente relacionada con esta dimensión.

De lo anterior se deduce que, en virtud de la interdependencia, la adaptación no sería un hecho aislado sino una función de los intercambios entre subsistemas. Así, la adaptación de un sistema estaría determinada por la posibilidad de reconocer lo que otros sistemas y subsistemas le podrían brindar y lo que cada uno podría ofrecer para atender a necesidades de los demás.

De otra parte, en un amplio marco de las ciencias del comportamiento, se destaca la literatura que vincula los procesos de adaptación con los procesos de organización (Gresoi y Diaconu, 2013; Rantakari, 2013; Szulanski y Jensen, 2006). La crítica a las perspectivas funcionalistas y conductuales relaciona la administración con el interés por la génesis y desarrollo de las estructuras organizativas (Stigliani y Ravasi, 2012; Girotto y Mundet, 2009; Piaget, 1989, 1983, 1979), desplazando el eje hacia la interacción entre los procesos organizadores y sus conexiones con la coordinación del todo organizado. En esta perspectiva, la adaptación emerge, fundamentalmente, en la concepción de su papel estructurante y organizador de la organización.

La preocupación por el origen y desarrollo de las estructuras es la razón del estructuralismo genético, que integra epistemologías de la biología del conocimiento y la psicología en el estudio de las funciones y estructuras de adaptación y de organización. Piaget et al. (2008) señalan que la organización, en sentido general, no es disociable de la adaptación y que la estabilidad no es sinónimo de inactividad sino, más bien, la manifestación de los procesos de cambio de las estructuras. De este modo la organización, entendida como función, es la acción del funcionamiento total sobre las subestructuras y la adaptación supone su transformación.

Para comprender a qué se refiere la adaptación de la organización-función es necesario diferenciar la adaptación estado de la adaptación como proceso. Es la primera acepción (estado) la que ha primado en los estudios del fenómeno adaptativo en los trabajos relacionados con la administración. Entre tanto, las condiciones de la adaptación como proceso inherente a la dinámica de la organización involucran los procesos de asimilación y acomodación (Piaget et al. 2008; Watzlawick, 2010, 2007), es decir, el cambio orientado por procesos de significación más que por la necesidad de responder a las presiones y demandas del entorno.

En tanto la adaptación deviene en las interacciones entre las experiencias, el contexto y la maduración, el mecanismo organizador de la organización son sus interacciones y lo que orienta la interacción es la comunicación (Marc y Picard, 1992). Así, en la 
interacción se hacen presentes las tensiones entre las estructuras e intereses que conviven dentro de la organización y la diversidad en sus mecanismos de asimilación.

Las tensiones se resuelven recurriendo a mecanismos de acomodación y/o de adaptación. En la acomodación la organización reacciona a las demandas del entorno y modifica sus estructuras y sus modos de interacción para solucionar dichas demandas en el corto plazo, lo que puede generar otras tensiones y el aislamiento entre sus unidades, es decir, podría favorecer la fragmentación.

En el caso de la adaptación, la organización interpreta el contexto de las demandas externas y utiliza sus estructuras y experiencias para modificarlo y desarrollarse, es decir, para posibilitar (permitir o promover) transformaciones que redunden en mayores niveles de complejización, autoorganización y desarrollo. Es, entonces, una organización creativa, transformadora y propositiva, no - primordialmente - reactiva a las demandas externas.

De lo anterior se deduce que la adaptación, en sentido estricto, emerge del equilibrio entre la asimilación y la acomodación, pero es cualitativamente diferente. En la adaptación se asiste a la complejización de las estructuras en redes de comunicaciones y significados, en tanto que en la acomodación la estructura se instrumentaliza en favor de la reacción rápida para atender a los requerimientos del exterior.

La tradición de la psicología social también se ha ocupado de la adaptación. En esta línea son referentes los trabajos de Pichón-Riveré (1999) cuando propone una concepción de salud en términos de adaptación activa a la realidad. Desde finales del siglo $\mathrm{XX}$ su trabajo ha sido retomado por investigadores que aplican la noción en estudios sobre la que han denominado "salud organizacional".

La psicología social en las organizaciones implica considerar cómo éstas moldean a los sujetos y cómo ellos las construyen mediante múltiples y complejos procesos y estructuras. En este sentido, se ocupa de las interacciones dirigidas a obtener cambios planificados que suponen el intercambio y la interdependencia entre las estructuras y las funciones. Esos intercambios e interdependencias implican, nuevamente, la acción a través de papeles o roles que juegan los sujetos en la organización.

Pichón Rivieré (1999) diferencia dos nociones: la adaptación activa y la adaptación pasiva. La primera atiende a las propias condiciones del sujeto, a sus capacidades intelectuales y emocionales para transformarse en la interacción con su medio y responder desde su propia condición a demandas y necesidades del entorno. En la adaptación pasiva el individuo se desconecta de su propia realidad y responde a estereotipos. Estas concepciones de adaptación también tienen alcance para la comprensión de la adaptación - activa o pasiva - de las organizaciones.

En el ámbito organizacional, la tendencia a explicar la adaptación de las organizaciones a partir de las metáforas biológicas incluyó los tres tipos o niveles de adaptación propuestos en el contexto de la teoría de la evolución: la morfológica, la fisiológica y la etológica. A lo anterior se suma que adaptación y adaptabilidad suelen utilizarse sin diferencia y se asimilan con capacidades adaptativas. En otros casos, hay términos que se utilizan en condiciones similares, como viabilidad, sostenibilidad, perdurabilidad, entre otros.

Algunos autores matizan las metáforas biológicas para intentar explicar el componente adaptativo de las organizaciones. Gresoi y Diaconou (2013) creen que la organización es un organismo adaptativo en permanente conexión con el ambiente, el cual influye en los parámetros de la organización. Señalan que en el campo de la gerencia organizacional la adaptabilidad se define a través de la capacidad para generar transformaciones en la organización, la creación de redes de trabajo para responder efectivamente a los cambios en el ambiente y mantenerse en éste. De este modo, un sistema cuyos parámetros responden a las condiciones del ambiente es, según los autores, un sistema adaptativo. 
En trabajos que datan de la segunda mitad del siglo XX hasta la actualidad, es evidente la coexistencia de dos tendencias: una línea de investigación que hace énfasis en la adaptación de la organización a las contingencias ambientales (March y Simon, 1977, 1958) y otra línea que propone el paradigma de la ecología de poblaciones, en el entendido de la adaptación como un proceso lento de reacción a los cambios del ambiente (Hannan y Freeman, 1984).

Una perspectiva tradicional de la adaptación organizacional se ancla en la clásica teoría de la contingencia, dependencia de recursos y la teoría de los costos de transacción con las múltiples formas de diseño de la organización para enfrentar los desafios de su supervivencia. Compatible con la ecología de poblaciones, propone que el ambiente selecciona características, mediante selección natural, a través de la reproducción y la duplicación.

Otros investigadores no consideran que haya conflicto entre adaptación organizacional y las perspectivas de la selección ambiental (natural). Las dos son complementarias (Levinthal, 1991; Astley y Van de Ven, 1983). Para Levinthal (1991) la adaptación es un ejercicio inteligente que también se conoce como aprendizaje y actúa como un balance de significados entre exploración y explotación organizacional.

Un tema central en la adaptación de las organizaciones es el equilibrio entre la exploración de nuevas posibilidades y la explotación de las certidumbres internas existentes (Holland, 1992). Una postura en la que las organizaciones deberían encontrar continuamente procesos de adaptación en relación con los procesos de conocimiento. Mantener el balance entre encontrar nuevas posibilidades y mejorar las rutinas operativas es esencial para que las organizaciones perduren y prosperen (March, 1991).

Para otros, la adaptación organizacional requiere la creación de variedad facilitada por el aprendizaje, pero el aprendizaje no se identifica con adaptación. La creación y transferencia de conocimientos serían los vehículos para la innovación y la cooperación.
Según March (1991) y Levinthal (1991), el conocimiento y el aprendizaje organizacional influyen sobre la adaptación de la organización a los rápidos cambios del entorno. Así, las organizaciones que poseen capacidades superiores para administrar el conocimiento tendrán más capacidad para adaptarse a la incertidumbre y la complejidad del entorno (Child y Gunter, 2001). Estos enfoques podrían resultar especialmente relevantes tratándose de organizaciones altamente diversas y de conocimiento, como las IES.

Otra visión vincula la adaptación con las crisis que enfrentan las organizaciones y establecen relaciones con los procesos de significación (Margulis y Sagan, 2013; Hage, Aiken y Marret, 1971). Las crisis requieren la reorganización de los procedimientos establecidos y estandarizados o la creación de nuevos significados para salir de ellas. En gran parte, los grupos y las organizaciones responden combinando comportamientos emergentes con rutinas del comportamiento organizacional (Soda y Zaheer, 2012).

En un cuestionamiento a la organización maquinal, Mintzberg (1991) aborda los problemas de adaptación como problemas de coordinación en la administración, que se convierten en problemas de adaptación en el nivel estratégico. La coordinación puede ser vista como el grado en el cual se conectan las partes de la estructura organizacional y las organizaciones pueden ser coordinadas de dos formas: por plan y por feedback. (Soda y Zaheer, 2012).

El plan se basa en cronogramas preestablecidos y programas dirigidos y estandarizados del funcionamiento de la organización en tanto que el feedback se centra en transmisión de nueva información para facilitar el acoplamiento mutuo de las partes. La prevalencia de cualquiera de las dos formas de coordinación impacta la manera como la administración desempeña sus funciones en las actividades cotidianas de la organización.

Claramente estos dos tipos de coordinación son constructos ideales. En realidad, las organizaciones complejas usan una mezcla de los dos. Sin embargo, 
es posible identificar las variables organizacionales asociadas con uno u otro mecanismo de coordinación (Hage, Aiken y Marret, 1971).

De otro lado, se intenta proponer una explicación sociológica para lo que tradicionalmente se ha descrito como fenómenos emergentes, relacionados con las crisis. Estos argumentos de lo que por mucho tiempo se ha llamado emergente, pueden ser explicados por: a) el incremento que se requiere para la coordinación durante las crisis, b) las condiciones que hacen que emerjan cambios en los patrones de comunicación interna, c) las consecuencias que los cambios de los patrones de comunicación tienen para la coordinación organizacional (Dynes y Aguirre, 2011).

Desde el punto de vista empresarial y productivo la adaptación se ha abordado en función de la competitividad, rentabilidad y la necesidad de responder a los cambios del ambiente o el entorno. Las restricciones que impone esta postura han llevado a que trabajos recientes busquen considerar el papel decisivo de la dirección en la adaptación organizativa y se destaque la reorganización interna como el mecanismo de respuesta a los cambios ambientales (Santos y García, 2006).

Cameron (1984) examina el papel del ambiente y de la administración en la adaptación de las organizaciones (cuadro 1). Este autor también examina las relaciones entre la innovación y la adaptación, y otros exploran el rol del marketing estratégico en la adaptación a los rápidos cambios en el entorno (Brown, 2012). La pregunta central es cómo las estructuras de la organización soportan el cambio a los ambientes externos y cómo ocurre éste. En búsqueda de respuestas, se utiliza el estudio de casos, también en el marco de la Teoría de la Contingencia. Adicionalmente, autores como Núñez, Gutiérrez y Carmona (2003) insisten en la prioridad de la información para la sobrevivencia de la Organización.

\section{Cuadro 1. El papel del ambiente y de la administración en la adaptación de las organizaciones}

\begin{tabular}{|l|l|l|}
\hline \multicolumn{1}{|c|}{ Aproximación } & \multicolumn{1}{|c|}{ Papel del ambiente } & \multicolumn{1}{c|}{ Papel de la administración } \\
\hline Ecología poblacional & Determinante & Ninguno o extremadamente bajo. \\
\hline Ciclos vitales & Prominente. Fuerzas evolutivas & Podrían alterar las fuerzas evolutivas. Incidencia baja \\
\hline Elección estratégica & $\begin{array}{l}\text { Rol importante. Equilibrio con el } \\
\text { rol de la administración }\end{array}$ & Importante. Equilibrio con el rol del medio ambiente. El equilibrio se desplaza hacia la gestión \\
\hline La acción simbólica & Muy bajo & $\begin{array}{l}\text { Determinante. Habilidad para crear, interpretar símbolos y las definiciones sociales. } \\
\text { Construcción de realidad }\end{array}$ \\
\hline
\end{tabular}

Fuente: construcción propia, con base en la propuesta de Cameron (1984).

Por otro lado, el Modelo de Sistemas Viables propuesto por Beer $(1996,1995,1992)$ y descrito por Achterbergh y Vriens (2010), relaciona las funciones requeridas para la adaptación con la identidad y la misión de la organización. Este modelo describe las funciones necesarias y suficientes para la viabilidad de la organización, articula las funciones con las relaciones y provee un conjunto de normas para el diagnóstico de la viabilidad organizacional. La viabilidad, en estos términos, es la capacidad de la organización para sobrevivir.

Por su parte, Baard, Rench y Kozlowski (2013) destacan el papel de la adaptación y su aplicación en diferentes niveles de la organización. Su taxonomía se refiere a tres niveles del desempeño adaptativo: el individual, el de los equipos de trabajo y la adaptación de la organización. La investigación se ha concentrado básicamente en el nivel micro y en el nivel 
meso, siendo la investigación de la adaptación en el nivel organizacional aún muy difusa, por lo que sugieren la profundización y trabajo específico en este nivel, y la posibilidad de explorar la aplicabilidad de las taxonomías de los niveles micro y meso, en el nivel macro de la adaptación organizacional.

El tema también se ha explorado en relación con situaciones puntuales. Joseph y Ocasio (2012) analizan cómo la arquitectura organizacional de una empresa multinegocio afecta la adaptación de las unidades de negocio que la constituyen. Usan un análisis inductivo de los sistemas de gobierno entre 1951 y 2001 para examinar la integración y atención de las unidades, dentro y a través de los canales de gobierno. Concluyen que la adaptación es la consecuencia de elaboradas relaciones entre la parte administrativa y las unidades de negocio.

El tema de la adaptación de las organizaciones también se aborda en relación con su rol social. En el marco de una teoría sistémico-interpretativa, Fuenmayor (2001) desarrolla un planteamiento que vincula el origen de la organización con su papel en la sociedad. Según este punto de vista, en las sociedades modernas una organización surge como un intento para solucionar un problema social. Este objetivo se concreta en la misión de la organización, el cual expresa una interpretación acerca de ese problema social y su solución.

Pero la interpretación puede no coincidir con el requerimiento social supuesto en esa interpretación y la consecuencia es el fracaso de la organización (Fuenmayor, 2001:44). En otras palabras, la adaptación y la pertinencia social de la organización trascienden su orientación o capacidad para la realización de los intereses de grupos sociales particulares, va más allá del aseguramiento de su rentabilidad y solvencia financiera.

El planteamiento de Fuenmayor comparte con el estructuralismo genético la relevancia que otorgan al origen en relación con la pertinencia social, por un lado, y la adaptación, por el otro. En cualquier caso, la relación de la génesis con el cambio en las estructuras tiene implicaciones que involucran procesos de aprendizaje y de desarrollo.

De manera menos directa el tema también ha merecido la atención de otros grupos académicos. La American Psychological Association (APA), en su Manual de Publicaciones, indica que la adaptación se refiere a la modificación de un material para que sea adecuado para un nuevo propósito (APA, 2010: 173). Esta idea pone el acento en los cambios sobre el material usado para un propósito, lo que podría ser extensivo a un instrumento o un procedimiento y, además, insinúa que este uso se aparta del objetivo original del material, el instrumento o el procedimiento. No se trataría, entonces, de una copia de algo, sino de desarrollos y construcciones a partir de algo. Una noción cercana a ciertas concepciones actuales de la innovación.

En lo que parece una nueva etapa de los supuestos del aprendizaje organizacional en boga durante la segunda mitad del siglo XX, la adaptación de las organizaciones también se ha vinculado con procesos de gestión de los conocimientos, una tendencia que se ha incrementado en el mundo de los negocios y que, desde una perspectiva educativa, resulta de la mayor relevancia. Más aún, cuando no es infrecuente que algunas universidades definan su misión en torno a procesos de gestión de conocimientos.

Para mejorar su adaptación a los acelerados cambios en el ambiente las organizaciones han implementado formas de gestión de conocimientos a fin de no cometer los mismos errores, resolver problemas en tiempos más cortos y estimular la innovación. En un intenso trabajo, Niu (2009) investigó los estadios de la gestión del conocimiento y describió cómo se obtiene, se organiza y se aplica, haciendo énfasis en sus efectos sobre la adaptación de la organización. La cuestión de cómo se obtiene, organiza y aplica el conocimiento representa, evidentemente, un núcleo de reflexión sobre procesos de adaptación en las instituciones de educación superior. 


\section{La adaptación y las instituciones de educación superior}

No abundan los trabajos que profundicen en los problemas de adaptación de las universidades, por lo que son significativos los aportes de algunos autores que han hecho referencia a temas relacionados.

Sporn (1999) examina el nivel de adaptabilidad de las organizaciones de educación superior en los Estados Unidos y Europa, desde la perspectiva de los factores socioeconómicos. Esta investigadora propone un enfoque de la adaptación en educación superior que incluye las estructuras de gobierno, de administración y el liderazgo. Utilizando el estudio de cuatro casos concluye que una organización académica ideal es abierta al cambio, con estructuras colegiadas de gobierno que proveen soporte a los procesos de adaptación. Destaca el papel de la administración, el liderazgo y el espíritu emprendedor.

La adaptación de las IES también puede leerse asociada a la calidad, lo que requiere un abordaje diferente al que se utilizaría en organizaciones de sectores productivos, comerciales, industriales o de servicios. Es indispensable una reflexión profunda acerca de la naturaleza de la noción de adaptación y sus formas de manifestarse, para poder emprender la operacionalización del concepto y construir indicadores que permitan comprender su naturaleza, sus manifestaciones y su evolución en el tiempo y el espacio (Misas, 2004).

Misas (2004), en una reflexión que desborda los límites de la universidad pública, plantea el problema en una perspectiva histórica. La universidad - como ícono de las IES - ha enfrentado permanentemente la tensión entre adaptarse al entorno o tratar de que el entorno se adapte a ella. La universidad ha vivido una tensión creciente acerca de la pertinencia de sus saberes y la forma de comunicarlos a la sociedad. Entender la adaptación de la universidad desde sus tensiones y conflictos históricos demanda mirarla y estudiarla en toda su complejidad y las relaciones que establece con sus entornos, con sus tensiones, contradicciones, interdependencias y complementariedades.

Desde este punto de vista, la universidad no puede basar su adaptación y pertinencia respondiendo a las demandas que le impone alguno de los actores sociales. La advertencia que se deriva de esta postura es trascendental: la universidad deja de ser pertinente cuando centra su formación en los procedimientos y no en los fundamentos: cuando su gestión busca responder a demandas externas, se comprometen su identidad y su misión.

Observamos como las concepciones de la adaptación de las organizaciones sociales han matizado las explicaciones centradas en la teoría de la evolución y han incorporado o construido constructos y estructuras conceptuales para entender un fenómeno que es común tanto a los procesos cognitivos como a los organizacionales. También, la importancia que desde algunos enfoques se atribuye a la administración para lograr la adaptación de las organizaciones sugiere la necesidad de problematizar este nivel de la responsabilidad administrativa, más allá de los desafíos que representan la supervivencia, la expansión o la rentabilidad de las apuestas académicas y formativas de las IES. El cuadro 2 recoge una síntesis de estas perspectivas.

La referencia directa a la adaptación de organizaciones de educación superior, que provienen especialmente de sectores de la academia, señala vacíos, pero también la conveniencia de construir problemas que las conecten con sus entornos de múltiples formas (Mora, 2018; Orozco, 2010) y permitan desarrollar líneas de reflexión y de investigación que den cuenta de las particularidades de las IES, a partir de la articulación de conceptos y la delimitación de sus campos de significación. 


\section{Cuadro 2. Visiones de la adaptación organizacional}

\begin{tabular}{|c|c|}
\hline Enfoque & Características \\
\hline Metafórico & $\begin{array}{l}\text { Utiliza metáforas biológicas para interpretar fenómenos sociales. Usa como referente la Teoría de la Evolución } \\
\text { para comprender la adaptación. Retoma los tres niveles de la adaptación biológica para describir adaptaciones } \\
\text { sociales: nivel morfológico (estructural), nivel fisiológico (funcional), nivel etológico (comportamental). } \\
\text { Aproximaciones investigativas basadas en el positivismo y métodos empírico-analíticos. }\end{array}$ \\
\hline Matices biológicos-sociales & $\begin{array}{l}\text { Entiende la organización como un organismo adaptativo, en conexión permanente con el medio ambiente. } \\
\text { Define, en una perspectiva administrativa, la adaptabilidad como capacidad para generar cambios en la } \\
\text { organización, la creación de redes de trabajo para responder a cambios en el entorno, buscando perdurabilidad. } \\
\text { Predominio de estrategias de conocimiento ancladas en postulados de las ciencias naturales. }\end{array}$ \\
\hline Complementariedad & $\begin{array}{l}\text { Puntos de vista que conciben la adaptación como aprendizaje y el aprendizaje como capacidad para actuar teniendo } \\
\text { en cuenta los resultados de acciones y experiencias anteriores. Aproximaciones de investigación que recogen } \\
\text { elementos del empirismo e incorporan componentes hermenéuticos. }\end{array}$ \\
\hline Variedad & $\begin{array}{l}\text { La adaptación como creación de diversidad en el comportamiento, facilitada por el aprendizaje y relaciones } \\
\text { basadas en la comunicación. La investigación tiende a incorporar la lógica y métodos de las ciencias sociales en } \\
\text { la construcción de problemas y los métodos para la recolección y análisis de la información. }\end{array}$ \\
\hline Reorganización & $\begin{array}{l}\text { La adaptación se asume como proceso de reorganización de procedimientos y patrones para resolver crisis } \\
\text { mediante la creación de nuevos niveles de significación. La investigación tiende a combinar métodos y enfoques, } \\
\text { con predominio del pragmatismo y modelos mixtos. }\end{array}$ \\
\hline Viabilidad & $\begin{array}{l}\text { La adaptación se asimila a viabilidad como conjunto de actividades encaminadas al fortalecimiento y el } \\
\text { desarrollo de la identidad y la misión de la organización. Combinación de enfoques de investigación con } \\
\text { predominio de los tipos cuantitativos, multinivel. }\end{array}$ \\
\hline Taxonómico & $\begin{array}{l}\text { Determinación y evaluación del desempeño adaptativo en función de tres niveles: micro (individual), meso (equipos } \\
\text { de trabajo) y macro (organizacional). Los estudios se han centrado en los dos primeros niveles. Predominio de } \\
\text { enfoques cuantitativos y correlacionales. }\end{array}$ \\
\hline Sistémico interpretativo & $\begin{array}{l}\text { Comprensión de la adaptación en función de los vínculos entre el origen y la pertinencia social de la organización. } \\
\text { Papel fundamental de la interpretación para alinear estas dos dimensiones. Consecuente con postulados } \\
\text { posmodernos privilegia rutas heurísticas, enfoques cualitativos y mixtos, Critical Management. }\end{array}$ \\
\hline Ajuste & $\begin{array}{l}\text { La adaptación como modificación de un material, dispositivo o procedimiento para adecuarlo a un nuevo } \\
\text { propósito. Cercano a concepciones y procesos de innovación. }\end{array}$ \\
\hline
\end{tabular}

Fuente: construcción propia. 


\section{Perspectiva de la universidad latinoamericana}

En un horizonte de análisis sobre la universidad latinoamericana, Scherz (1986) señala que los modelos emergentes de finales de siglo XX se desarrollan con la fuerte influencia de las lógicas de mercado y mirando la evolución de modelos como el norteamericano y europeo. Por su parte, Barnett (2013) subraya que la universidad siempre ha estado supeditada a los poderes dominantes; entenderlos daría luces para comprender el itinerario de la universidad latinoamericana. Por lo tanto, decisiones que afectan la orientación de las universidades de la región tendrían que leerse en el contexto de los intereses económicos y políticos y sus implicaciones sociales.

De otro lado, Orozco (2010) advierte que las IES deben enfrentar condiciones cambiantes que cuestionan la misión que están llamadas a cumplir. Los dilemas que enfrentan se deben a que muchas de las demandas que se hacen a la universidad superan su capacidad para responder. Enfoca la cuestión en la forma como se debería construir una universidad necesaria, capaz de identificar con claridad sus compromisos con el país y con la región, sin sacrificar su proyecto y misión, siendo pertinente y fiel a sí misma.

En una perspectiva similar, López y Casanova (2013) señalan cómo las políticas derivadas del neoliberalismo han impactado fuertemente a la educación superior en América Latina y propiciaron la irrupción de lo privado en la esfera pública. La privatización no se limitó a la progresiva influencia de inversores en la educación superior, sino que ésta fue asimilando códigos y principios de lo privado en el ámbito del servicio público, lo que derivó, también, en el traslado de formas de gobierno de la empresa a la administración de la educación pública y privada.

Un punto de vista complementario concibe la universidad, independientemente de su condición pública o privada, como un conjunto estructurado de participantes en torno a un núcleo de compromisos que reflejan, en un momento determinado del tiempo, la correlación de fuerzas entre los diferentes agentes que la componen y las fuerzas del entorno. De conformidad con lo anterior, las tensiones internas y externas plantean desafíos a la forma como se organiza y responde la universidad. La organización de la universidad y sus formas de gestión deben ser construidas en función del proyecto académico que se tenga, no de las presiones externas; así, a medida que ese proyecto sufre cambios morfológicos, la estructura organizativa y las prácticas de gestión deben modificarse para interactuar internamente y con el entorno (Misas, 2004; Gómez, 2004; 2000).

Desde otra perspectiva algunos autores resaltan el desafío de adaptación que tienen las universidades en entornos cambiantes e inestables (Brunner, 2011; Brunner y Uribe, 2007; Cameron, 1984) por lo que simultáneamente se deben resolver problemas de costo, calidad, efectividad y acceso (Orozco 2010; Gumport y Sporn, 1999). En consecuencia, el desafío consistiría en cómo adaptarse a un contexto calificado como turbulento, particularmente en América Latina.

Finalmente, desde diversas perspectivas parece existir acuerdo en que muchos modelos de administración general y de los procesos académicos en las IES, así como de políticas relacionadas con la calidad, tienen como referentes o emulan modelos básicamente provenientes de las universidades anglosajonas en sus vertientes británica y norteamericana y/o responden a demandas de organismos internacionales. La pregunta sobre cómo esta tendencia podría reflejarse o impactar en aspectos como la pertinencia social y la construcción de indicadores de gestión contextualizada, no es irrelevante.

\section{Discusión}

El ejercicio heurístico adelantado mediante la revisión sistemática de literatura focalizada ha permitido establecer múltiples perspectivas de la adaptación y delimitar el concepto asociado con las responsabilidades de la administración, particularmente de las organizaciones de educación superior. Los hallazgos permiten 
delinear escenarios posibles para la investigación y la evaluación de las interacciones entre el concepto y las prácticas administrativas en las IES privadas.

Por su naturaleza, la institución de educación superior sería el tipo de organización en el que se esperaría una fuerte articulación entre teoría y práctica, no sólo en la gestión de los procesos académicos y científicos, sino en los aspectos generales de su administración. La falta o débil unidad en los propósitos, marcos y estilos de gestión entre los niveles general y específico podría redundar en la fragmentación de la organización y la inconsistencia entre lo expuesto como parte de su proyecto y las prácticas cotidianas de los actores educativos en sus interacciones internas y con el entorno. Es éste un supuesto que da cuenta de la relevancia de tener claros y alineados los principios filosóficos, teóricos y conceptuales que se traduzcan en modos de ser, de hacer y de valorar las acciones cotidianas.

La revisión también ha permitido corroborar que, aunque se asume como deseable y necesario que las instituciones se adapten, no se parte de referentes teóricos claros desde los cuales se asignen responsabilidades y se diseñen estrategias, sino que, con frecuencia, la adaptación se asume como una definición, más o menos amplia, vinculada a gestión del cambio, manejo de conflictos y casi exclusivamente con el propósito de dar respuesta a las demandas del entorno. Además, se identifica que muchas de las definiciones carecen de un sustento sólido epistemológico, por lo que no se observa la conexión lógica entre conceptos que nutren una teoría.

En algunos casos podría transitarse de definiciones a constructos que buscarían aportar a la explicación, entendimiento o intervención para responder a demandas y entornos cambiantes. En este sentido, el tiempo de respuesta se considera corto y el objetivo es la supervivencia y la perdurabilidad de la organización.

Plantear un marco para la problematización de las interacciones entre la adaptación y la administración de las IES requiere no sólo cuestionar lo que se entiende por adaptación, sino que supondría, también, algún nivel de reflexión sobre el significado y el sentido que comporta administrar este tipo específico de organización. Igualmente, exigiría la contextualización de los conceptos por parte de los generadores de política pública y los diseñadores de sistemas e indicadores de calidad de la educación superior.

El diseño de los escenarios posibles 'para la investigación incluye, inicialmente tres referentes centrales. El primero, el marco de las interacciones entre la administración y el medio ambiente, el segundo, los postulados del estructuralismo genético, y el tercero, el marco de la pertinencia social de las IES.

En relación con los roles de la administración y del medio ambiente en la adaptación, la visión incorpora la toma de decisiones como un factor fundamental que moviliza recursos para pasar de la ecología poblacional y las metáforas a los procesos de significación. En este tránsito se pueden generar condiciones y posibilidades para consolidar organizaciones simbólicas, consecuentes con un tipo de organización que trabaja, produce y transforma símbolos.

Para construir un campo de problemas la pregunta más general desde esta perspectiva podría ser, ¿en qué aproximación y bajo qué lógica (de la ecología poblacional, los ciclos vitales, la elección estratégica o la acción simbólica) regula la universidad sus interacciones y sus recursos? ¿cuáles son los mecanismos que utiliza la administración para hacerlo? En adelante, podría analizarse la coherencia de la lógica en uso con los marcos filosóficos y la misión de la universidad.

Es la complejidad y solidez epistemológica del estructuralismo genético la que brinda el terreno más fértil para formular interrogantes de fondo y construir un complejo escenario para analizar, comprender y transformar los modos de articular la administración con la adaptación en los niveles micro, meso y macro de las IES. Dado que el nivel macro es el menos estudiado y que es el objeto de este trabajo, los cuestionamientos principales se refieren a él. 
La construcción de un cuerpo de preguntas para configurar un sistema de investigación desde este enfoque supone la comprensión de los conceptos, su lugar en la teoría y, especialmente, la interacción entre ellos cuando se proyectan al ámbito de la organización. Un trabajo sugerente y seductor que invita a identificar y construir relaciones en torno a:

1. La transformación inherente a la adaptación se manifiesta en el origen y el desarrollo de estructuras cada vez más complejas. ¿El origen de las estructuras universitarias responde a procesos de desarrollo o a la adopción de modelos de estructuras organizacionales ancladas en el mundo empresarial, diseñadas para ser competentes y perdurables en un mundo altamente competitivo y mercantilizado? ¿El cambio de estructuras se expresa en la creatividad y desarrollo de las IES o en su burocratización? 2. A partir de la distinción entre la adaptación como estado y como proceso, ¿cuál privilegian las IES y los órganos de control? ¿Cómo las decisiones y las interacciones - mecanismo organizador de la organización - aportan a procesos de autoevaluación, interpretación y construcción de realidades, desde las IES?

3. Las tensiones internas y con el entorno se resuelven a través de los mecanismos de asimilación, acomodación y adaptación, ¿cuáles son las características de estos mecanismos reguladores e integradores en las IES? ¿Cuáles sus implicaciones en decisiones que involucran la asignación de responsabilidades, recursos, medios y formas de interactuar con los entornos y grupos de interés? ¿Cómo repercuten en la forma y los órganos de gobierno de la universidad?

4. En sentido estricto la adaptación surge del equilibrio entre la asimilación y la acomodación, ¿cómo se entiende y se expresa en prácticas científicas, académicas, pedagógicas y administrativas este equilibrio? ¿Cómo afecta los procesos de rendición de cuentas y la participación?
En el marco sistémico interpretativo, el rol social se encuentra fuertemente vinculado a la adaptación de las organizaciones. Tres cuestiones centrales harían posible una discusión y profundización inicial del tema en las IES:

1. El origen de la organización se ancla en la interpretación de su papel en la sociedad. ¿Cómo y en qué han cambiado las condiciones, necesidades y contexto de las IES y cómo se evidencia en su misión, organización y formas de interacción? ¿Es el entorno el que ha determinado los cambios o las IES han contribuido a comprender y resolver problemas sociales vinculados a su razón de ser?

2. La organización logra aportar a la comprensión y solución de problemas sociales mediante su interpretación. Para que esto ocurra, debe haber concordancia entre el requerimiento social y la propuesta de la organización. ¿Cómo, quiénes y bajo que supuestos y medios interpretan las IES su entorno cercano y lejano? ¿Cómo se involucran los órganos de toma de decisiones en ello?

3. La coherencia entre las condiciones de origen y la pertinencia social pueden condicionar la adaptación y esto implica procesos de aprendizaje y desarrollo, ¿corresponden los cambios en las estructuras y las conexiones a los problemas o a necesidades de reorganización institucional?

En conclusión, el campo de la investigación sobre las relaciones entre la administración de las IES está en construcción y demanda, de un lado, recurrir a la teoría y de otro, la articulación de visiones multidisciplinares y la conexión de los niveles de decisión de las instituciones y del Estado. El riesgo de la falta de precisión y de referentes teóricos que iluminen y permitan evaluar las prácticas es que, entre otras cosas, se asuman como reales y/o válidas condiciones y hechos que no lo son, por ejemplo, enunciar como adaptación procesos o estados que no pasan de la acomodación, con mayores o menores niveles de eficiencia en la asimilación. 


\section{Referencias}

Achterbergh, J., D. Vriens (2010), Organizations. Social systems conducting experiments, Londres, Springer.

American Psychological Association (2010), Manual de Publicaciones [ $3^{\circ}$. Ed. en español], Bogotá, El Manual Moderno.

Astley, W.G. y H. Van de Ven (1983), "Central perspectives and debates in organization theory", Administrative Science Quarterly, vol. 28, núm. 2, junio, pp. 245-273.

Baard, S., T. Rench y S. Kozlowski (2013), "Perfomance adaptation: a theoretical integration and review", fournal of Management, <http://jom.sagepub.com/content/ear ly/2013/05/24/0149206313488210> [Consulta: junio de 2014].

Barnett,R.(2013),ImaginingtheUniversity,Londres, Routledge.

Borrero, A. (1984), "Administración y estructuras académicas universitarias", en Simposio Permanente sobre la Universidad. Segundo Seminario General, 1983-1984, Bogotá, Ascun-Icfes.

Brown, S. M. (2012), "Organizational adaptation to the rapidly changing external environment: a case study of strategic marketing at Notre Dame College in Ohio", Education Doctoral Theses (Paper 46), Boston, Boston Northeastern University.

Brunner, J. J.(2011), “Gobernanza universitaria: tipología, dinámicas y tendencias", Revista de Educación, mayoagosto, pp. 137-159.

Brunner, J. J. y D. Uribe (2007), "Mercados universitarios: los nuevos escenarios de la educación superior", Informe final de Proyecto FODECyT 1050138, Chile.

Cameron, K. S. (1984), "Organizational Adaptation and Higher Education", Journal of Higher Education, vol. 55, núm. 2, marzo-abril.

Cerda, H. (1991), Elementos de la investigación, Bogotá, Editorial El Buho.

Cherryholmes, C. H. (1992), "Notes on pragmatism and scientific realism”, Educational Research, vol. 21, núm. 6, august-september, pp. 13-17.

Child, J. y R. Gunter (2001), "Organizations unfettered: organizational form in an information intensive economy", The Academy of Management fournal, vol. 44, núm. 6, pp. 1135-1148.

Centro Cochrane Iberoamericano (2012), Manual Cochrane de Revisiones Sistemáticas de Intervenciones [versión 5.1.0, actualizada en marzo de 2011], Barcelona, Centro Cochrane Iberoamericano, <http://www.cochrane. es/?q=es/node/269> [Consulta: septiembre de 2014].

Dynes, R. y B. E. Aguirre (2011), ”Organizational adaptation to crises: mechanisms of coordination and structural change", Crisis Management, 2, pp. 320-325.

Eichholz, J.C. (2015), Capacidad adaptativa, Bogotá, Ediciones de la $\mathrm{U}$.

Fuenmayor, R. (2001), Interpretando organizaciones. Una teoría sistémico interpretativa de las organizaciones, Venezuela, Universidad de los Andes.

Girotto, M. y J. Mundet (2009), "Strategic languag and micro-practices influence on strategy performance: a discursive approach to strategic planning process in higher education sector", 6th International Critical Management Studies Conference, Warwick, Warwick Business School, julio.

Gómez, V. (2004), Gobierno y gobernabilidad en las universidades públicas, Bogotá, Universidad Nacional de Colombia.

Gómez. V. (2000), Cuatro temas críticos de la educación superior en Colombia, Bogotá, Ascun/Universidad Nacional Alfaomega.

Gresoi, S. G. y A. Diaconou (2013), "Human resource management in support of improving the adaptability of the Romanian companies", Revista Română de Statistică, Supliment Trim II, pp. 155-160.

Gumport, P. y B. Sporn (1999), Institutional adaptation: demands for management reform and university administration, Stanford, National Center for Postsecondary Improvement, <http://www.stanford.edu/group/ ncpi/documents/pdfs/1-07_adaptation.pdf > [Consulta: abril de 2011].

Hage, J., M. Aiken y C. Marret (1971), "Organizational structure and communication", American Sociological Review, vol. 36, núm 5, pp. 360-371. 
Hannan, M. y J. Freeman (1984), "Structural inertia and organizational change", American Sociological Review, vol. 49, núm. 2, pp. 149-164.

Hernández-Sampieri, R., C. Fernández-Collado y P. Baptista (2006), Metodología de la investigación [4 Ed.], México, McGraw Hill.

Holland, J. (1992), Adaptation in natural and artificial systems, Cambridge, MIT Press.

Joseph, J. y W. Ocasio, (2012), "Architecture, attention and adaptation in the multibusiness firm: General Electric from 1951 to 2001", Strategic Management Fournal, vol. 33, pp. 633-660, DOI: 10.1002/ smj.1971.

Levinthal, D. A. (1991), "Organizational adaptation and environmental selection-interrelated process of change", Organization Science, vol. 2, núm. 1, pp. 140-145.

López García,J. y H. Casanova Cardiel(2013), "Educación superior en México: los límites del neoliberalismo (2000-2010)", Linhas Críticas, vol. 19, núm. 38, eneroabril, pp. 109-128, <http://www.redalyc.org/articulo. oa?id=19352631 1008> [Consulta: junio, 2016].

Marc, E. y D. Picard (1989), La interacción social, Barcelona, Editorial Paidós.

March, A. S. (1991), "Exploration and exploitation in organizational Learning", Organizational Science, (1), pp. 71-87.

March, J. G. y H.A. Simon (1958), Organizations, Nueva York, Wiley Press (version en español, 1977, Teoría de la Organización, Barcelona, Editorial Ariel).

Margulis, L. y D. Sagan (2013), Microcosmos, Buenos Aires, Tusquets Editores.

Merriam, S. (1988), Investigación con estudios de caso en educación: un enfoque cualitativo, San Francisco, Jossey Bass.

Mintzberg, H. (1991), Mintzberg y la dirección, Madrid, Ediciones Díaz de Santos S.A.

Misas, G. (2004), La educación superior en Colombia. Análisis y estrategias para su desarrollo, Bogotá, Universidad Nacional de Colombia/Unibiblos.

Mora, J. G. (2018), "Universidades: mitos, modas y tendencias", Revista Iberoamericana de Educación Superior (RIES), vol. IX, núm. 24, pp. 3-16, <http://dx.doi.org/10.22201/ iisue.20072872e2018.24.259> [Consulta: mayo de 2018].

Moustakas, C. (1990), Heuristic research. Design, methodology and applications, Londres, Sage Publications.

Niu, K-H. (2009), "Knowledge management and organizational adaptation: a theoretical framework of mediation and moderation", Review of Business Research, vol. 9, núm. 1, pp. 8-19.

Nuñez, M., I. Gutiérrez y S. Carmona (2003), "Informar para sobrevivir", Cuadernos de Economía y Dirección de la Empresa, 14, pp. 5-28.

Orozco, L. E. (2010), "Calidad académica y relevancia social de la educación superior en América Latina", Revista Iberoamericana de Educación Superior (RIES), vol. I, núm. 1, pp. 24-36, <https://ries.universia.net/article/ viewFile/31/90> [Consulta: mayo de 2015].

Parsons, T. (1951), "El sistema social", <https:// teoriasuno.files.wordpress.com/2013/08/el-sistemasocial-talcott-parsons.pdf $>$ [Consulta: febrero de 2014]

Piaget, J. (1989), La psicología de la inteligencia, Barcelona, Editorial Crítica.

Piaget, J. (1983), Adaptación vital y psicología de la inteligencia, México, Siglo XXI Editores.

Piaget, J. (1979), Psicología y epistemología, Barcelona, Ariel Editores.

Piaget, J., P. Osterrieth, J. Nuttin, F. Bresson, Ch. Marx y F. Meyer (2008), Los procesos de adaptación, Buenos Aires, Paidós.

Pichón-Riveré, E. (1999), Del psicoanálisis a la psicología social, Buenos Aires, Editorial Galerna.

Rantakari, H. (2013), Governing adaptation, Marshall School of Business, University of Southern California, <https://msbfile03.usc.edu/digitalmeasures / rantakar/intellcont/Adaptation_revision-1.pdf> [Consulta: marzo de 2014].

Rossman, G. y B. Willson (1985), "Numbers and words: combining qualitative and quantitative methods in a single large-scale evaluation", Evaluation Review, vol. 9, núm. 5, pp. 627-643. 
Salk, J. (1983), Anatomy of reality, Nueva York, Columbia University Press.

Santos, V. y T. García (2006), "El papel de la dirección en la adaptación organizativa: un estudio para empresas españolas", Management International, Summer, vol. 10, núm. 4, pp. 29-68.

Scherz, L. (1986), "La Universidad del año 2000: entre Napoleón y Humboldt", Nueva Sociedad, 84, pp. 91-99, julio-agosto, 1986.

Smith, B. y J. Wandel, (2006), "Adaptation, adaptative capacity and vulnerability", Global Environmental Change, 16, pp. 282-292.

Soda, G. y A. Zaheer (2012), "A network perspective on organizational architecture: perfomance effects of the interplay of formal and informal organization", Strategic Management fournal, 33, pp. 751-771, DOI: 10.1002/smj 1966.
Sporn, B. (1999), Adaptative University structures: an analysis of adaptation to socioeconomic environments of US and European universities, Londres, Jessica Kingsley Publishers.

Stigliani, I. y D. Ravasi (2012), "Organizing thoughts and connecting brains: material practices and the transition from individual to group-level prospective sensemaking", Academy of Management fournal, vol. 55, núm. 5, pp. 1232-1259, <http://cb!.doi.org/10.5465/ amj.2010.0890> [Consulta: abril de 2014]

Szulanski, G. y R. Jensen (2006), "Presumptive adaptation and the effectiveness of knowledge transfer", Strategic Management Fournal, 27, pp. 937-957, DOI: 10.1002/ smj.551.

Watzlawick, P. [ed.] (2010), La realidad inventada: cómo sabemos lo que creemos saber, Barcelona, Editorial Gedisa.

Watzllawick, P. (2007), Cambio: formación y solución de los problemas humanos, Barcelona editorial Herder. 